035.

\section{Use of Seven-Digit Telephone Numbers to Access EMS in a Community Served by 911}

David Sheldon, MD, * William Fales, MD, Dena Smith, EMT-P, Myriam Korstanje, $B A$

Michigan State University, Kalamazoo Center for Medical Studies, Kalamazoo, Michigan USA

Purpose: To determine patient outcome and caller rationale when requesting EMS using a seven-digit telephone number (SDTN).

Methods: All requests for EMS received from 01 January to 31 March 1995 by the ambulance service dispatch centers through a SDTN and identified as potentially life threatening (Priority 1) were selected. Cases from nursing homes and other health-care facilities were excluded. Patient outcomes were determined by reviewing medical records. Using callback telephone numbers, a structured telephone interview was conducted to determine the rationale for using a SDTN (instead of 911).

Results: A total of 2,371 Priority-1 requests were received. Approximately $1,778(75 \%)$ of these requests were received through 911 . Of the requests, $86(3.6 \%)$ were received through a SDTN and met inclusion criteria. Of these patients, two $(2.3 \%)$ presented in cardiac arrest and failed to survive, 40 $(46.5 \%)$ were discharged from the ED, $44(51.2 \%)$ were admitted to the hospital, $24(27.9 \%)$ were admitted to critical-care units and four expired in the hospital. Data from 40 callers were available through a structured telephone interview. Of those interviewed, two $(5.0 \%)$ were unaware that 911 existed, seven $(17.5 \%)$ did not want a lights-and-siren response, 25 $(62.5 \%)$ were ambulance-service subscribers, $32(80.0 \%)$ had a seven-digit telephone-number sticker, three $(7.5 \%)$ had the telephone number programed into a speed dial, four $(10.0 \%)$ called based on an advertisement, and three $(7.5 \%)$ were dissatisfied with a competing service.

Conclusion: In our system, requests for EMS in potentially lifethreatening emergencies through a SDTN were infrequent. However, these cases were of high acuity as reflected by the high overall hospital and critical-care admission rates and mortality rate. Factors influencing the use of a SDTN were varied.
039.

\section{The Utility of a Two-Tiered Prehospital Provider-Initiated Trauma Alert System}

C. Keith Stone, $M D,{ }^{*} 1$ J. Stephan Stapczynski, $M D,{ }^{1}$ Stephen H. Thomas, $M D,^{2}$ Patrick Muck ${ }^{I}$

1. Department of Emergency Medicine, University of Kentucky, Lexington, Kentucky USA

2. Department of Emergency Services, Massachusetts General Hospital/Harvard Medical School, Boston, Massachusetts USA

Purpose: To study the utility of a two-tiered trauma system that is initiated by prehospital care providers.

Methods: Trauma registry data for trauma alerts initiated by ground transport paramedics were collected over 13 months. The trauma alert system studied is initiated by prehospital personnel based on mechanism of injury and clinical assessment. A "trauma alert" brings the trauma team to meet the patient on arrival to ED. A "trauma alert red" additionally holds open an OR for 30 minutes. Data collected included age, type of trauma, injury severity score (ISS), ED disposition to the OR and mortality. Data were analyzed using Student's $t$-test and chi-square test with alpha set at 0.05 .

Results: One hundred fifteen patients were studied with $\mathbf{5 3}$ trauma alerts and 63 trauma alert reds. No differences were noted in age $(31.8 \pm 17.8$ vs $33.5 \pm 13.8$ years $)$, ISS $(20.2 \pm 9.8$ vs $14.7 \pm 17.9$ ), ED disposition to the OR ( $25 \%$ vs $35 \%$ ) or mortality $(30 \%$ vs $21 \%)$, trauma alert versus trauma alert red. There was a significant difference in the percentage of both blunt $(66 \%$ vs $27 \%)$ and penetrating $(33 \%$ vs $73 \%)$ trauma alert versus trauma alert red.

Conclusion: The trauma alert red, when initiated by prehospital care providers, ties up OR resources for patients who have the same rate of OR usage, injury severity and mortality as standard trauma alert patients. There appears to be no advantage to a two-tiered prehospital provider initiated trauma alert system. 